\title{
Credit financing for deteriorating imperfect quality items with allowable shortages
}

\author{
Aditi Khanna ${ }^{\mathrm{a}}$, Mandeep Mittal ${ }^{\mathrm{b}}$, Prerna Gautam ${ }^{\mathrm{a}}$ and Chandra K. Jaggi ${ }^{\mathrm{a}^{*}}$
}

\begin{abstract}
${ }^{a}$ Department of Operational Research, Faculty of Mathematical Sciences, New Academic Block, University of Delhi, Delhi-110007, India
${ }^{b}$ Department of Industrial and Management Engineering, Hanyang University, Ansan, Gyeonggi-do, 426 791, South Korea ${ }^{b}$ Amity School of Engineering \& Technology, 580-Delhi PalamVihar Road, Bijwasan, New Delhi-1 10061, India

C H R O N I C L E

Article history:

Received June 25, 2015

Received in revised format:

September 2, 2015

Accepted September 4, 2015

Available online

September 52015

Keywords:

Inventory

Imperfect quality items

Deterioration

Shortages

Credit financing

The outset of new technologies, systems and applications in manufacturing sector has no doubt lighten up our workload, yet the chance causes of variation in production system cannot be eliminated completely. Every produced/ordered lot may have some fraction of defectives which may vary from process to process. In addition the situation is more susceptible when the items are deteriorating in nature. However, the defective items can be secluded from the good quality lot through a careful inspection process. Thus, a screening process is obligatory in Moreover, in order to survive in the current global markets, credit financing has been proven a very influential promotional tool to attract new customers and a good inducement policy for the retailers. Keeping this scenario in mind, the present paper investigates an inventory model for a retailer dealing with imperfect quality deteriorating items under permissible delay in payments. Shortages are allowed and fully backlogged. This model jointly optimizes the order quantity and shortages by maximizing the expected total profit. A mathematical model is developed to depict this scenario. Results have been validated with the help of numerical example. Comprehensive sensitivity analysis has also been presented.
\end{abstract}

\section{Introduction}

The classical economic order quantity (EOQ) models, although practical and functional are based on assumptions that are restrictive and utopian, due to which they do not cater many industries today. In practical scenario, the inventory model should incorporate certain features which exhibit the real inventory situation. Out of many factors, deterioration is one such factor which should not be ignored, because there is inventory loss by deterioration and the inventory value is dependent on the product value at the time of evaluation. Generally, deterioration is defined as damage, spoilage, decay, obsolescence, evaporation, pilferage, etc. that results in decreasing the usefulness of the original one. For items such as steel, hardware, glassware and toys, the rate of deterioration is low in each case; there is little need for considering deterioration in the determination of the economic lot size. However, some

* Corresponding author.

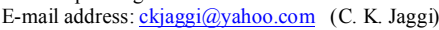

C 2016 Growing Science Ltd. All rights reserved. doi: $10.5267 /$ j.ds1.2015.9.001
(C) 2016 Growing Science Ltd. All rights reserved. 
items such as food items, pharmaceuticals, fashion goods, chemicals, blood, alcohol, gasoline and radioactive chemical deteriorate rapidly over time. At first, Ghare and Scharader (1963) presented an EOQ model for deteriorating items assuming exponential decay. Covert and Philip (1973) extended the model with the assumption of Weibull distribution deterioration. Thereafter, several interesting papers related to deterioration appeared in different journals such as Dave and Patel (1981), Hollier and Mark (1983), Sachan (1984) etc. which were summarized by Raafat et al. (1991). Chung and Ting (1994), Hariga and Benkherouf (1994) also contributed in the field of deterioration.

Another common unrealistic assumption in using the EOQ model is that all units produced are of good quality. But practically it is difficult to produce or purchase items with $100 \%$ good quality. Thus, the inspection of lot becomes indispensable in most of the organizations. By considering this very fact, researchers developed various EPQ/EOQ models with defective items. Porteus (1986) incorporated the effect of defective items into the basic economic order quantity model. Rosenblatt and Lee (1986) assumed that the time between the beginnings of the production run; i.e., the in-control state; until the process goes out of control is exponential and that defective items can be reworked instantaneously at a cost and they concluded that the presence of defective products motivates smaller lot sizes. Salameh and Jaber (2000) extended the work accomplished for imperfect quality items under random yield and developed economic order quantity which contradicts with the findings of Rosenblatt and Lee (1986) that the economic lot size quantity tends to decrease as the average percentage of imperfect quality items increases. Konstantaras (2006) looked at the issue of non-shortages in model with proportional imperfect quality, when the proportion of the imperfect is a random variable. Maddah and Jaber (2008) rectified a flaw in an economic order quantity model with unreliable supply, characterized by a random fraction of imperfect quality items and a screening process. Recently, Maddeh et al. (2010) proposed an improved practical approach for preventing shortages during screening period and they suggested that the order is placed when the inventory level is just enough to cover the demand during the screening period. Then, the demand during the screening period of an order is met from the inventory of "previous" order. Further, when the items are deteriorating in nature, then the inspection of lot becomes pertinent. Recently, Jaggi et al. (2013) developed a model for imperfect deteriorating items with time dependent demand under inflationary conditions.

Further, it is generally assumed that payment will be made to the supplier for the goods immediately after receiving the consignment. However, in day-to-day dealing, it is found that the supplier allows a certain fixed period to settle the account. During this period, no interest is charged by the supplier, but beyond this period interest is charged under certain terms and conditions agreed upon, since inventories are usually financed through debt or equity. In case of debt financing, it is often a short-term financing. Thus, interest paid here is nothing but the cost of capital or opportunity cost is significant. Also, shortterm loans can be thought of as having been taken from the supplier on the expiry of credit period. However, before the account has to be settled, the customer can sell the goods and continues to accumulate revenue and earn interest instead of paying the overdraft that is necessary if the supplier requires settlement of the account after replenishment. Interest earned can be thought of as a return on investment, since the money generated through revenue can be ploughed back into the business. Therefore, it makes economic sense for the customer to delay the settlement of the replenishment account up to the last day of the credit period allowed by the supplier. Kingsman (1983) explored the effects of payment rules on ordering and stocking in purchasing. Goyal (1985) and Davis and Gaither (1985) developed economic order quantity under conditions of permissible delay in payments. Aggarwal and Jaggi (1995) extended Goyal (1985) model by considering the point that if the credit period is less than the cycle length, the customer continues to accumulate revenue and earns interest on it for the rest of the period in the cycle, from the stock remaining beyond the credit period. Further Chu et al. (1998) proved the piecewise convexity of the total cost function of Aggarwal and Jaggi (1995). Since then, many research papers have appeared in different journals, which have been summarized by Soni et al. (2010). Chung and Huang (2006) developed EOQ model with imperfect quality and a permissible period for non-deteriorating items. 
All of the above-mentioned models assume that shortages are not permitted to occur. Never-the less, in many practical situations, stock out is unavoidable due to various uncertainties. Therefore, the occurrence of shortages in inventory is a reasonable event. Thus, in practical dealing we have to deal with shortages. Hariga (1995) and Chakrabarti and Chaudhari (1997) developed models for deteriorating items with time-varying and linear trend in demand. Wee et al. (2007) developed optimal inventory model for items with imperfect quality and shortage backordering. Chang and Ho (2010) considered imperfect quality items and shortage backordering, and obtained closed-form expressions. Further, Jaggi et al. (2013b) discussed economic ordering policies for defective items with shortages and trade-credit. However, they assumed that the items are non-deteriorating in nature.

Recently, Haidar et al. (2014) developed a model considering effect of deterioration on the instantaneous replenishment model with imperfect quality items in which they assume that $100 \%$ screening is conducted immediately after an order is received. Then at the end of screening process, imperfect items are removed from inventory and sold as a single batch at a discounted price. They developed the model for both the cases i.e., with shortages and without shortages.

The present paper provides an approach to study the problem of a retailer dealing with imperfect quality deteriorating items under permissible delay in payments. Shortages are allowed and fully backlogged. It is assumed that screening rate is more than demand rate. This assumption helps one to meet his demand and backorders parallel to screening process, out of the items which are of perfect quality. The present model jointly optimizes the order quantity and shortages by maximizing the retailer's expected total profit. A comprehensive sensitivity analysis has also been performed to give some important managerial insights.

\section{Assumptions and Notations}

\subsection{Assumptions}

The following assumptions are used to develop the model:

1.The demand rate is known, constant, and continuous.

2.Each lot received contains a random proportion of defective items, $\alpha$, with a known p.d.f. $f(\alpha)$.

3.Shortages are allowed. Backlogged demand is met right after screening is completed.

4.A constant fraction $\theta$ of the on-hand inventory deteriorates per unit time.

5.The supplier provides a fixed credit period $M$ to settle the accounts to the retailer.

6.The screening and demand proceeds simultaneously, but the screening rate $(\lambda)$ is greater than demand rate $(D), \lambda>\mathrm{D}$.

7.The defective items are independent of deterioration.

\subsection{Notations}

The following notations are used to develop the model:

\begin{tabular}{ll}
\hline$k$ & ordering cost \\
$c$ & retailer's purchasing price \\
$h$ & holding cost per unit time \\
$\theta$ & proportion of the on-and inventory lost due to deterioration \\
$\alpha$ & average proportion of defective items \\
$\lambda$ & screening rate in units per unit time \\
$\beta$ & unit screening cost \\
$t_{s}$ & screening time \\
$Q$ & order quantity \\
$T$ & cycle length \\
\hline
\end{tabular}


$D \quad$ demand rate per unit time

$I_{e} \quad$ interest earned

$I_{p} \quad$ interest paid

$b \quad$ maximum backorder level allowed

$p \quad$ selling price per unit

$f(\alpha)$ Probability density function of $\alpha$

$E(\alpha)$ Expected value of $\alpha$, which is equal to $=\int_{a}^{b} \alpha f(\alpha) d \alpha, \quad 0<a<b<1$

$M \quad$ permissible delay in settling the accounts

$C_{2} \quad$ shortage cost per unit per unit time

$C_{s} \quad$ salvage cost per defective unit(unit selling price of imperfect quality items)

$I_{1}(t)$ Inventory level during the time interval $\left(0, t_{s}\right)$

$I_{2}(t)$ Inventory level during the time interval $\left(t_{s}, t_{1}\right)$

\section{Mathematical Model}

We contemplate the problem of a lot size $Q$ delivered instantaneously to a retailer, with a purchasing price $c$ per unit and an ordering cost $K$. It is assumed that each lot received contains a random proportion of defective items, $\alpha$ with a known probability density function, $f(\alpha)$. Upon receiving each lot, a $100 \%$ screening process is conducted at a screening rate $\lambda$ per unit time. The defective items found are kept in stock and sold at the end of the cycle as a single batch at a discounted price of $C_{s}$ per unit, where $C_{s}<c$. Let $D$ be the demand rate per unit time, $\theta$ the proportion of the on-hand inventory lost per unit time due to deterioration, $t_{s}$ the screening time per cycle, $t_{s}=Q / \lambda$, and $T$ the cycle length. It is assumed that the backlogged demand is met right after screening is completed. In addition, let $b$ be the backorder level, and $t_{2}$ the time to build a backorder level of $b$ units at the rate $D, t_{2}=b / D$. During time interval $\left(0, t_{s}\right)$ the inventory level, $I_{1}(t)$ decreases due to the effect of both demand and deterioration. At time $t_{s}$, the screening process is completed and the inventory level drops by the expected number of defective items found in the lot, $\alpha Q$ and by backorders, $b$, the backordered demand which is satisfied from the inventory. During the time interval $\left(t_{s}, t_{1}\right)$, the inventory level $I_{2}(t)$ decreases due to demand and deterioration, and reaches zero at time $t_{1}$. At this time, the backordered demand starts building at the demand rate $D$, until the beginning of a new cycle, when a new lot of size $Q$ is received. The demand is still backordered shortly after receiving the new lot, until screening of the new lot is conducted at time $t_{s}$, when the backordered demand is delivered instantaneously.

Let $I_{1}(t)$ be the inventory level at time $\left(0 \leq t \leq t_{s}\right)$

Differential Equation for period, $\left(0, t_{s}\right)$ is given by:

$$
\frac{d I_{1}(t)}{d t}+\theta I_{1}(t)=-D, 0 \leq t \leq t_{s}
$$

The solution of the above differential equation along with the boundary condition, $t=0, I_{1}(0)=Q$ is,

$$
I_{1}(t)=\frac{D}{\theta}\left(e^{-\theta t}-1\right)+Q e^{-\theta t}
$$

Since after the screening process, the number of defective items at time $t_{s}$ is $\alpha Q$ and the backorders is $b$. Further, the effective inventory level at $t=t_{s}$, after removing the defective items and backorders is given by:

$$
I_{e f f .\left(t_{s}\right)}=Q e^{-\theta t_{s}}+\frac{D}{\theta}\left(e^{-\theta t_{s}}-1\right)-\alpha Q-b
$$


Now, let $I_{2}(t)$ be the inventory level at any time $\left(t_{s} \leq t \leq t_{1}\right)$

Differential equation for period $\left(t_{s}, t_{1}\right)$ is given by:

$$
\frac{d I_{2}(t)}{d t}+\theta I_{2}(t)=-D_{0}, t_{s} \leq t \leq t_{1}
$$

The solution of the above differential equation with boundary condition, $t=t_{s}, I_{2}\left(t_{s}\right)=I_{e f f}$. $\left(t_{s}\right)$ is given by:

$$
I_{2}(t)=\frac{D}{\theta}\left(e^{-\theta t}-1\right)+Q e^{-\theta t}-\alpha Q e^{\theta\left(t_{s}-t\right)}-b e^{\theta\left(t_{s}-t\right)}
$$

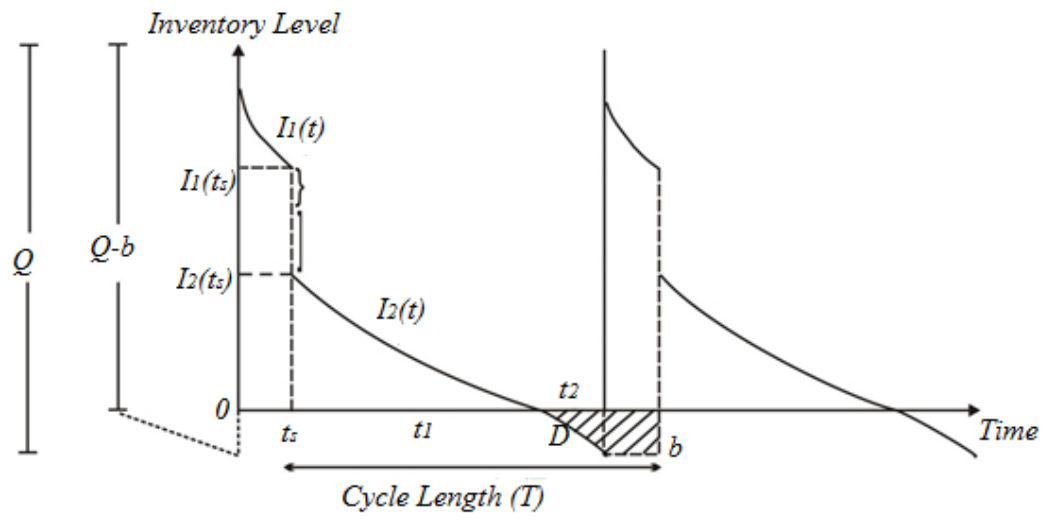

Fig. 1. Graphical representation of inventory level over time $T$

We will solve the equation for $t_{1}$ with condition $t=t_{1},\left(I_{2}\right) t_{1}=0$

$$
\begin{aligned}
& t_{1}=\frac{1}{\theta}\left[\log \left(\frac{D}{\theta}+Q-\alpha Q e^{\theta t_{s}}-b e^{\theta t_{s}}\right)-\log \left(\frac{D}{\theta}\right)\right] \\
& T=t_{1}+t_{2},
\end{aligned}
$$

where, $t_{2}=b / D$

The present model has been developed under the condition of permissible delay in payments, therefore, depending upon the credit period, there would be three distinct possible cases for retailer's total profit $\pi_{j}, j=1,2,3$, viz.

Case 1: $0 \leq M \leq t_{s}$

Case 2: $t_{s} \leq M \leq t_{1}$

Case 3: $t_{1} \leq M \leq t_{2}$

Since, the retailer's total profit consists of the following components:

$$
\begin{array}{r}
\pi_{j}=\text { Sales Revenue }- \text { Ordering cost }- \text { Screening Cost }- \text { Deterioration cost } \\
- \text { Holding Cost }- \text { shortage cost }+ \text { Interest Earned }- \text { Interest Paid } .
\end{array}
$$


Therefore, various individual components are evaluated as follows:

1. Total sales revenue is the sum of revenue generated by the demand meet during the time period $(0, T)$ and sale of imperfect quality items is

2. Ordering cost $=K$

3. Screening cost $=\beta Q$

4. Shortage cost $=C_{2} b\left(t_{2}+2 t_{s}\right)$, where $\mathrm{b}$ is the maximum permissible shortage.

5. Deterioration cost $=c(Q-D T)$

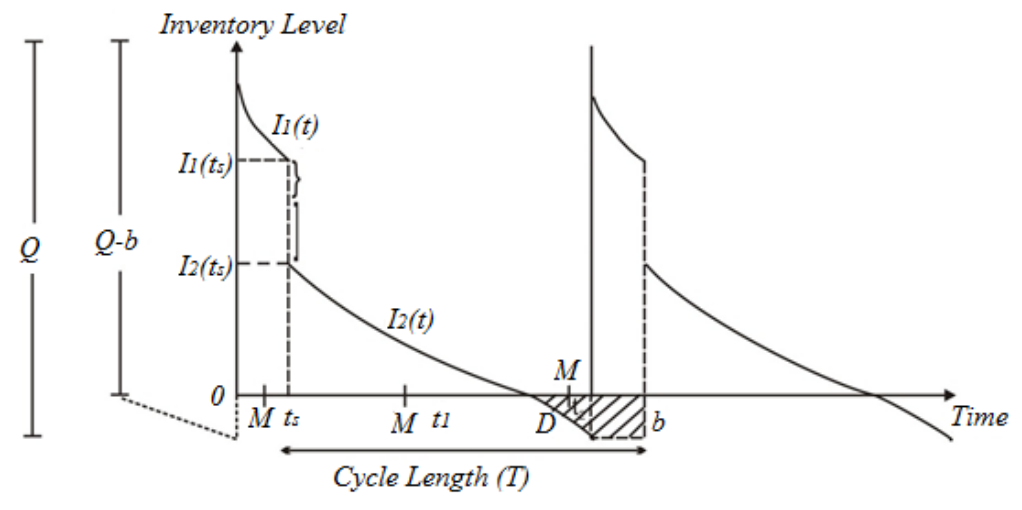

Fig. 2. Graphical representation of inventory system over time $T$

Holding cost during the time period 0 to $t_{s}$ and $t_{s}$ to $t_{1}$

$$
\begin{aligned}
=h & {\left[\int_{0}^{t_{s}} I_{1}(t) d t+\int_{t_{s}}^{t_{1}} I_{2}(t) d t\right] } \\
& \text { Holding Cost }=h\left[\left[\frac{D}{\theta}\left(\frac{-1}{\theta}\left(e^{-\theta t_{s}}-1\right)-t_{s}\right)-Q\left(\frac{1}{\theta}\left(e^{-\theta t_{s}}-1\right)\right]+\left[\frac { D } { \theta } \left(\frac { - 1 } { \theta } \left(e^{-\theta t_{1}}-\right.\right.\right.\right.\right. \\
& \left.\left.\left.\left.e^{-\theta t_{s}}\right)-\left(t_{1}-t_{s}\right)\right)-\frac{Q}{\theta}\left(e^{-\theta t_{1}}-e^{-\theta t_{s}}\right)-\frac{\alpha Q}{\theta}\left(1-e^{\theta\left(t_{s}-t_{1}\right)}\right)-\frac{b}{\theta}\left(1-e^{\theta\left(t_{s}-t_{1}\right)}\right)\right]\right]
\end{aligned}
$$

To determine interest earned and interest payable, there will be three cases:

Case 1: $0 \leq M \leq t_{s}$

In this case, one can earn interest on revenue generated from the sales up to $M$. Although one has to settle the account at $M$, one has to arrange money at some specified rate of interest in order to get his remaining stocks financed for the period $M$ to $T$.

$$
\begin{aligned}
& \text { Interest Earned }=p I_{e} \int_{0}^{M} D t d t=p I_{e} \frac{D M^{2}}{2} \\
& \text { Interest payable }=c I_{p}\left[\int_{M}^{t_{s}} I_{1}(t) d t+\int_{t_{s}}^{t_{1}} I_{2}(t) d t\right] \\
& =c I_{p}\left[\left\{\begin{array}{c}
\frac{D}{\theta}\left(\frac{-1}{\theta}\left(e^{-\theta t_{s}}-e^{-\theta M}\right)-\left(t_{s}-M\right)\right) \\
\frac{Q}{\theta}\left(e^{-\theta t_{s}}-e^{-\theta M}\right)
\end{array}\right\}+\left\{\frac{D}{\theta}\left(\frac{-1}{\theta}\left(e^{-\theta t_{1}}-e^{-\theta t_{s}}\right)-\left(t_{1}-t_{s}\right)\right)-\right.\right. \\
& \left.\left.\frac{Q}{\theta}\left(e^{-\theta t_{1}}-e^{-\theta t_{s}}\right)-\frac{\alpha Q}{\theta}\left(1-e^{\theta\left(t_{s}-t_{1}\right)}\right)-\frac{b}{\theta}\left(1-e^{\theta\left(t_{s}-t_{1}\right)}\right)\right\}\right]
\end{aligned}
$$


Substitute the values from Eqs. (9-16) in Eq. (8), the total profit for case $1, \pi_{1}(Q, b)$ becomes

$$
\begin{aligned}
& \pi_{1}(Q, b)=p(1-\alpha) Q+C_{s} \alpha Q-K-\beta Q-C_{2} b\left(t_{2}+2 t_{s}\right)-c(Q-D T)- \\
& h\left[\left[\frac{D}{\theta}\left(\frac{-1}{\theta}\left(e^{-\theta t_{s}}-1\right)-t_{s}\right)-Q\left(\frac{1}{\theta}\left(e^{-\theta t_{s}}-1\right)\right]+\left[\frac{D}{\theta}\left(\frac{-1}{\theta}\left(e^{-\theta t_{1}}-e^{-\theta t_{s}}\right)-\left(t_{1}-t_{s}\right)\right)-\right.\right.\right. \\
& \left.\left.\frac{Q}{\theta}\left(e^{-\theta t_{1}}-e^{-\theta t_{s}}\right)-\frac{\alpha Q}{\theta}\left(1-e^{\theta\left(t_{s}-t_{1}\right)}\right)-\frac{b}{\theta}\left(1-e^{\theta\left(t_{s}-t_{1}\right)}\right)\right]\right]+p I_{e} \frac{D M^{2}}{2}- \\
& c I_{p}\left[\left\{\begin{array}{c}
\frac{D}{\theta}\left(\frac{-1}{\theta}\left(e^{-\theta t_{s}}-e^{-\theta M}\right)-\left(t_{s}-M\right)\right)- \\
\frac{Q}{\theta}\left(e^{-\theta t_{s}}-e^{-\theta M}\right)
\end{array}\right\}+\left\{\frac{D}{\theta}\left(\frac{-1}{\theta}\left(e^{-\theta t_{1}}-e^{-\theta t_{s}}\right)-\left(t_{1}-t_{s}\right)\right)-\right.\right. \\
& \left.\left.\frac{Q}{\theta}\left(e^{-\theta t_{1}}-e^{-\theta t_{s}}\right)-\frac{\alpha Q}{\theta}\left(1-e^{\theta\left(t_{s}-t_{1}\right)}\right)-\frac{b}{\theta}\left(1-e^{\theta\left(t_{s}-t_{1}\right)}\right)\right\}\right]
\end{aligned}
$$

Case 2: $t_{s} \leq M \leq t_{1}$

In this case one can earn interest on revenue generated from sales up to $M$, one will also earn interest for the shortages which are met during $\left(M-t_{s}\right)$ and due to the sale of defective items during $\left(M-t_{s}\right)$.

$$
\begin{aligned}
& \text { Interest Earned }=p I_{e}\left[\int_{0}^{M} D t d t+b\left[\left(M-t_{s}\right)\right]+C_{s} \alpha Q I_{e}\left(M-t_{s}\right)\right. \\
& =p I_{e}\left[\frac{D M^{2}}{2}+b\left(M-t_{s}\right)\right]+C_{s} \alpha Q I_{e}\left(M-t_{s}\right) \\
& \quad \text { Interest payable }=c I_{p}\left[\int_{M}^{t_{1}} I_{2}(t) d t\right] \\
& =c I_{p}\left[\frac{D}{\theta}\left(\frac{-1}{\theta}\left(e^{-\theta t_{1}}-e^{-\theta M}\right)-\left(t_{1}-M\right)\right)-\frac{Q}{\theta}\left(e^{-\theta t_{1}}-e^{-\theta M}\right)-\frac{\alpha Q}{\theta}\left(1-e^{\theta\left(M-t_{1}\right)}\right)-\right. \\
& \left.\frac{b}{\theta}\left(1-e^{\theta\left(M-t_{1}\right)}\right)\right]
\end{aligned}
$$

Substitute the values from Eqs. (9)-(14), Eq. (18) and Eq. (19) in Eq. (8), the total profit for case 2, $\pi_{2}(Q, b)$ becomes

$$
\begin{aligned}
& \pi_{2}(Q, b)=p(1-\alpha) Q+C_{s} \alpha Q-K-\beta Q-C_{2} b\left(t_{2}+2 t_{s}\right)-c(Q-D T)- \\
& h\left[\left[\frac{D}{\theta}\left(\frac{-1}{\theta}\left(e^{-\theta t_{s}}-1\right)-t_{s}\right)-Q\left(\frac{1}{\theta}\left(e^{-\theta t_{s}}-1\right)\right)\right]+\left[\frac{D}{\theta}\left(\frac{-1}{\theta}\left(e^{-\theta t_{1}}-e^{-\theta t_{s}}\right)-\left(t_{1}-t_{s}\right)\right)-\right.\right. \\
& \left.\left.\frac{Q}{\theta}\left(e^{-\theta t_{1}}-e^{-\theta t_{s}}\right)-\frac{\alpha Q}{\theta}\left(1-e^{\theta\left(t_{s}-t_{1}\right)}\right)-\frac{b}{\theta}\left(1-e^{\theta\left(t_{s}-t_{1}\right)}\right)\right]\right]+p I_{e}\left[\frac{D M^{2}}{2}+b\left(M-t_{s}\right)\right]+ \\
& C_{s} \alpha Q I_{e}\left(M-t_{s}\right)-c I_{p}\left[\frac{D}{\theta}\left(\frac{-1}{\theta}\left(e^{-\theta t_{1}}-e^{-\theta M}\right)-\left(t_{1}-M\right)\right)-\frac{Q}{\theta}\left(e^{-\theta t_{1}}-e^{-\theta M}\right)-\frac{\alpha Q}{\theta}(1-\right. \\
& \left.\left.e^{\theta\left(M-t_{1}\right)}\right)-\frac{b}{\theta}\left(1-e^{\theta\left(M-t_{1}\right)}\right)\right]
\end{aligned}
$$

Case 3: $t_{1} \leq M \leq T$

In this case we discuss the situation when inventory cycle is greater than or equal to permissible delay. Thus, in this scenario no interest is payable by the retailer and in addition to interest earned in previous case one is earning interest for the demand met for the time period $\left(M-t_{1}\right)$

$$
\begin{aligned}
& \text { Interest Earned }=p I_{e} \int_{0}^{t_{1}} D t d t+p I_{e}\left[D t_{1}\right]\left[M-t_{1}\right]+p I_{e} b\left[M-t_{s}\right]+C_{s} \alpha Q I_{e}\left[M-t_{s}\right] \\
& =p I_{e}\left(\left(\frac{D t_{1}^{2}}{2}\right)+\left[D t_{1}\right]\left[M-t_{1}\right]+b\left[M-t_{s}\right]\right)+C_{s} \alpha Q I_{e}\left[M-t_{s}\right]
\end{aligned}
$$


Substitute the values from Eqs. (9)-(14), Eq. (21) and (22) in Eq. (8), the total profit for case 3, $\pi_{3}(Q, b)$ becomes

$$
\begin{aligned}
& \pi_{3}(Q, b)=p(1-\alpha) Q+C_{s} \alpha Q-K-\beta Q-C_{2} b\left(t_{2}+2 t_{s}\right)-c(Q-D T)- \\
& h\left[\left[\frac{D}{\theta}\left(\frac{-1}{\theta}\left(e^{-\theta t_{s}}-1\right)-t_{s}\right)-Q\left(\frac{1}{\theta}\left(e^{-\theta t_{s}}-1\right)\right)\right]+\left[\frac{D}{\theta}\left(\frac{-1}{\theta}\left(e^{-\theta t_{1}}-e^{-\theta t_{s}}\right)-\left(t_{1}-t_{s}\right)\right)-\right.\right. \\
& \left.\left.\frac{Q}{\theta}\left(e^{-\theta t_{1}}-e^{-\theta t_{s}}\right)-\frac{\alpha Q}{\theta}\left(1-e^{\theta\left(t_{s}-t_{1}\right)}\right)-\frac{b}{\theta}\left(1-e^{\theta\left(t_{s}-t_{1}\right)}\right)\right]\right]+p I_{e}\left(\left(\frac{D t_{1}^{2}}{2}\right)+\left[D t_{1}\right][M-\right. \\
& \left.\left.t_{1}\right]+b\left[M-t_{s}\right]\right)+C_{s} \alpha Q I_{e}\left[M-t_{s}\right]
\end{aligned}
$$

\section{Case 4: $T \leq M$}

In this case, all the expressions for interest earned and paid coincide with that of previous case.

Hence, effectively we have three different cases for the retailer's total profit per cycle, $\pi(Q, b)$, which can be expressed as

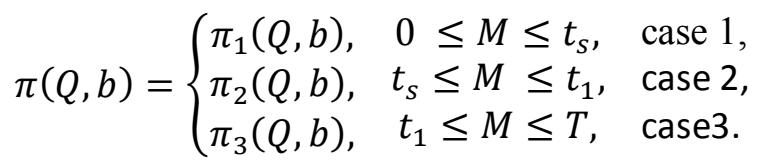

To determine the expected total profit per unit time, we apply renewal reward theorem Ross, (1996) as $\alpha$ is a random variable with known probability density function, $f(\alpha)$ and get the expected total profit per unit time for different cases which follows:

$$
\begin{aligned}
& E\left[\pi^{T}(Q, b)\right]=E\left[\frac{\pi(Q, b)}{T}\right]=\frac{E[\pi(Q, b)]}{E[T]}, \\
& \pi(Q, b)=\left\{\begin{array}{llll}
E\left[\pi_{1}^{T}(Q, b)\right]=E\left[\pi_{1}(Q, b)\right] / E[T], & 0 \leq M \leq t_{s}, & \text { case } 1, & (26 \mathrm{a}) \\
E\left[\pi_{2}^{T}(Q, b)\right]=E\left[\pi_{2}(Q, b)\right] / E[T], & t_{s} \leq M \leq t_{1}, & \text { case } 2, & (26 \mathrm{~b}) \\
E\left[\pi_{3}^{T}(Q, b)\right]=E\left[\pi_{3}(Q, b)\right] / E[T] . & t_{1} \leq M \leq T, & \text { case3. } & \text { (26 c) }
\end{array}\right.
\end{aligned}
$$

where,

$$
\begin{aligned}
& E\left[\pi_{1}(Q, b)\right]=p(1-E[\alpha]) Q+C_{s} E[\alpha] Q-K-\beta Q-C_{2} b\left(t_{2}+2 t_{s}\right)-c(Q-D T)- \\
& h\left[\left[\frac{D}{\theta}\left(\frac{-1}{\theta}\left(e^{-\theta t_{s}}-1\right)-t_{s}\right)-Q\left(\frac{1}{\theta}\left(e^{-\theta t_{s}}-1\right)\right)\right]+\left[\frac{D}{\theta}\left(\frac{-1}{\theta}\left(e^{-\theta t_{1}}-e^{-\theta t_{s}}\right)-\left(t_{1}-t_{s}\right)\right)-\right.\right. \\
& \left.\left.\frac{Q}{\theta}\left(e^{-\theta t_{1}}-e^{-\theta t_{s}}\right)-\frac{E[\alpha] Q}{\theta}\left(1-e^{\theta\left(t_{s}-t_{1}\right)}\right)-\frac{b}{\theta}\left(1-e^{\theta\left(t_{s}-t_{1}\right)}\right)\right]\right]+p I_{e} \frac{D M^{2}}{2}- \\
& c I_{p}\left[\left\{\begin{array}{c}
\left.\frac{D}{\theta}\left(\frac{-1}{\theta}\left(e^{-\theta t_{s}}-e^{-\theta M}\right)-\left(t_{s}-M\right)\right)-\right) \\
\frac{Q}{\theta}\left(e^{-\theta t_{s}}-e^{-\theta M}\right)
\end{array}\right\}+\left\{\frac{D}{\theta}\left(\frac{-1}{\theta}\left(e^{-\theta t_{1}}-e^{-\theta t_{s}}\right)-\left(t_{1}-t_{s}\right)\right)-\right.\right. \\
& \left.\left.\frac{Q}{\theta}\left(e^{-\theta t_{1}}-e^{-\theta t_{s}}\right)-\frac{E[\alpha] Q}{\theta}\left(1-e^{\theta\left(t_{s}-t_{1}\right)}\right)-\frac{b}{\theta}\left(1-e^{\theta\left(t_{s}-t_{1}\right)}\right)\right\}\right] \\
& E\left[\pi_{2}(Q, b)\right]=p(1-E[\alpha]) Q+C_{s} E[\alpha] Q-K-\beta Q-C_{2} b\left(t_{2}+2 t_{s}\right)-c(Q-D T)- \\
& h\left[\left[\frac{D}{\theta}\left(\frac{-1}{\theta}\left(e^{-\theta t_{s}}-1\right)-t_{s}\right)-Q\left(\frac{1}{\theta}\left(e^{-\theta t_{s}}-1\right)\right)\right]+\left[\frac{D}{\theta}\left(\frac{-1}{\theta}\left(e^{-\theta t_{1}}-e^{-\theta t_{s}}\right)-\left(t_{1}-t_{s}\right)\right)-\right.\right. \\
& \left.\left.\frac{Q}{\theta}\left(e^{-\theta t_{1}}-e^{-\theta t_{s}}\right)-\frac{E[\alpha] Q}{\theta}\left(1-e^{\theta\left(t_{s}-t_{1}\right)}\right)-\frac{b}{\theta}\left(1-e^{\theta\left(t_{s}-t_{1}\right)}\right)\right]\right]+p I_{e}\left[\frac{D M^{2}}{2}+b\left(M-t_{s}\right)\right]+
\end{aligned}
$$




$$
\begin{aligned}
C_{S} E[\alpha] Q I_{e}\left(M-t_{s}\right) & -c I_{p}\left[\frac{D}{\theta}\left(\frac{-1}{\theta}\left(e^{-\theta t_{1}}-e^{-\theta M}\right)-\left(t_{1}-M\right)\right)-\frac{Q}{\theta}\left(e^{-\theta t_{1}}-e^{-\theta M}\right)-\right. \\
\frac{E[\alpha] Q}{\theta}\left(1-e^{\theta\left(M-t_{1}\right)}\right)- & \left.\frac{b}{\theta}\left(1-e^{\theta\left(M-t_{1}\right)}\right)\right] \\
E\left[\pi_{3}(Q, b)\right]= & p(1-E[\alpha]) Q+C_{s} E[\alpha] Q-K-\beta Q-C_{2} b\left(t_{2}+2 t_{s}\right)-c(Q-D T) \\
& -h\left[\frac{D}{\theta}\left(\frac{-1}{\theta}\left(e^{-\theta t_{s}}-1\right)-t_{s}\right)-Q\left(\frac{1}{\theta}\left(e^{-\theta t_{s}}-1\right)\right)\right] \\
& +\left[\frac{D}{\theta}\left(\frac{-1}{\theta}\left(e^{-\theta t_{1}}-e^{-\theta t_{s}}\right)-\left(t_{1}-t_{s}\right)\right)-\frac{Q}{\theta}\left(e^{-\theta t_{1}}-e^{-\theta t_{s}}\right)\right. \\
& \left.\left.-\frac{E[\alpha] Q}{\theta}\left(1-e^{\theta\left(t_{s}-t_{1}\right)}\right)-\frac{b}{\theta}\left(1-e^{\theta\left(t_{s}-t_{1}\right)}\right)\right]\right] \\
& +p I_{e}\left(\left(\frac{D t_{1}{ }^{2}}{2}\right)+\left[D t_{1}\right]\left[M-t_{1}\right]+b\left[M-t_{s}\right]\right)+C_{s} E[\alpha] Q I_{e}\left[M-t_{s}\right]
\end{aligned}
$$

and, $E[T]=\frac{(1-E[\alpha]) Q}{D}$.

\section{Solution Procedure}

Our objective is to find the optimal values of $Q$ and $b$ which maximize the total profit function, $E\left[\pi^{T}(Q, b)\right]$, therefore, the necessary conditions for $E\left[\pi^{T}(Q, b)\right]$ to be optimal are $\frac{\partial E\left[\pi^{T}(Q, b)\right]}{\partial Q}=0$ and $\frac{\partial E\left[\pi^{T}(Q, b)\right]}{\partial b}=0$ which follows as case wise:

Case 1: $0 \leq M \leq t_{s}$

The optimal values of $Q=Q_{1}$ (say) and $b=b_{1}$ (say), which maximizes, $E\left[\pi_{1}^{T}(Q, b)\right]$ can be obtained by solving the Eq. (26a),

$$
\begin{aligned}
& X_{11}-X_{12}-X_{15}+X_{16}+X_{17}+X_{18}+X_{20}=0 \\
& X_{31}-X_{32}-X_{33}=0
\end{aligned}
$$

All $X_{i j}(i=1,2,3$ and $j=0,1, \ldots, 8)$ are elaborated in Appendix A.

Case 2: $t_{s} \leq M \leq t_{1}$

The optimal values of $Q=Q_{2}$ (say) and $b=b_{2}$ (say), which maximizes, $E\left[\pi_{2}^{T}(Q, b)\right]$, can be obtained by solving Eq. (26b),

$$
\begin{aligned}
& Y_{11}-Y_{12}-Y_{13}=0 \\
& Y_{21}-Y_{22}=0
\end{aligned}
$$

All $Y_{i j}(i=1,2$ and $j=1,2,3)$ are elaborated in Appendix B.

Case 3: $t_{1} \leq M \leq t_{2}$

The optimal values of $Q=Q_{3}$ (say) and $b=b_{3}$ (say), which maximizes, $E\left[\pi_{3}^{T}(Q, b)\right]$, can be obtained by solving Eq. (26c),

$$
\begin{aligned}
& Z_{11}+Z_{12}=0 \\
& Z_{21}+Z_{22}=0
\end{aligned}
$$

All $Z_{i j}(i=1,2$ and $j=1,2)$ are elaborated in Appendix C. 
Solutions are given in Appendix A, B and C.

Further, to prove the concavity of the expected profit function, the following sufficient conditions must be satisfied:

$$
\begin{gathered}
\left(\frac{\partial^{2} E\left[\pi^{T}(Q, b)\right]}{\partial Q \partial b}\right)^{2}-\left(\frac{\partial^{2} E\left[\pi^{T}(Q, b)\right]}{\partial Q^{2}}\right)\left(\frac{\partial^{2} E\left[\pi^{T}(Q, b)\right]}{\partial b^{2}}\right) \leq 0, \text { and } \\
\frac{\partial^{2} E\left[\pi^{T}(Q, b)\right]}{\partial Q^{2}} \leq 0, \frac{\partial^{2} E\left[\pi^{T}(Q, b)\right]}{\partial b^{2}} \leq 0
\end{gathered}
$$

All the second order derivatives have been calculated in Appendix D. Due to the complexity of these derivatives, it becomes difficult to prove the concavity mathematically; so the concavity of all the expected profit functions have been established graphically and graphs for different scenarios are shown in Figures below.

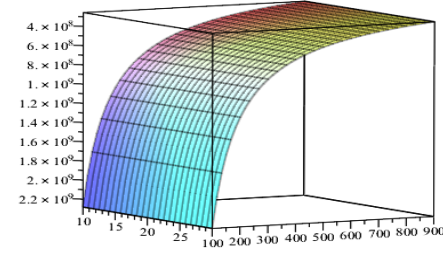

Fig. 3. Concavity of expected total profit function (case 1)

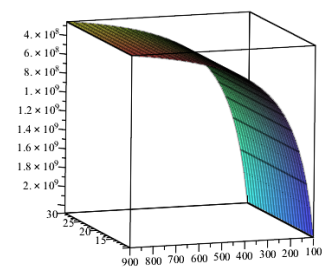

Fig. 4. Concavity of expected total profit function (case 2)

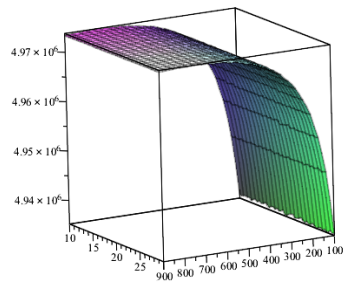

Fig. 5. Concavity of expected total profit function (case 3)

Now, in order to find the optimal values of $Q^{*}$ and $b^{*}$, which maximizes the retailer's expected profit, we propose the following algorithm:

\section{Algorithm}

1. Determine $Q^{*}=Q_{1}$ (say) and $b=b_{1}$ (say) from equation (30) and (31). Now using the value of $Q$ and $b$ calculate the value of $t_{1}$ and $T$ from equation (6) and (7). If $0 \leq M \leq t_{s}$ the expected total profit can be obtained from equation (26 a) else set $E\left[\pi_{1}(Q, b)\right]=0$.

2. Determine $Q^{*}=Q_{2}$ (say) and $b=b_{2}$ (say) from equation (30) and (31). Now using the value of $Q$ and $b$ calculate the value of $t_{1}$ and $T$ from equation (6) and (7). If $t_{s} \leq M \leq t_{1}$ the expected total profit can be obtained from equation (26 b) else set $E\left[\pi_{2}(Q, b)\right]=0$.

3. Determine $Q^{*}=Q_{3}$ (say) and $b=b_{3}$ (say) from equation (30) and (31). Now using the value of $Q$ and $b$ calculate the value of $t_{1}$ and $T$ from equation (6) and (7). If $t_{1} \leq M \leq T$ the expected total profit can be obtained from equation (26 c) else set $E\left[\pi_{3}(Q, b)\right]=0$.

4. Compare the calculated expected profit for case 1,2, and 3 and select the optimal values of $Q$ and $b$ associated with the maximum expected profit.

\section{Numerical Example}

Example 1. An example is devised to validate the model with the following data.

$D=50000$ units/year, $K=\$ 100$ per cycle, $c=\$ 25 /$ unit, $p=\$ 50 /$ unit, $h=\$ 10 /$ unit $/$ year, $\theta=0.05$,

$\alpha=0.01, \lambda=175200$ units/year, $\beta=\$ 0.5 /$ unit, $M=0.001$ year, $I_{e}=0.10 /$ year, $I_{p}=0.15 /$ year,

$C_{s}=\$ 20 /$ unit, and $C_{2}=\$ 15 /$ year.

Using the proposed algorithm results are obtained as follows: the optimal order level $Q^{*}=858$ units, the backorder level $b^{*}=22$ units, and the expected total profit $E\left[\pi^{T}(Q, b)\right]=\$ 1,198,021$. 
Example 2. An example is devised to validate the model with the following data.

$D=50000$ units/year, $K=\$ 100$ per cycle, $c=\$ 25 /$ unit, $p=\$ 50 /$ unit, $h=\$ 10 /$ unit $/$ year, $\theta=0.05$, $\alpha=0.01, \lambda=175200$ units/year, $\beta=\$ 0.5 /$ unit, $M=0.023$ year, $I_{e}=0.10 /$ year, $I_{p}=0.15 /$ year, $C_{s}=\$ 20 /$ unit, and $C_{2}=\$ 15 /$ year.

Using the proposed algorithm results are obtained as follows: the optimal order level $Q^{*}=830$ units, the backorder level $b^{*}=35$ units, and the expected total profit $E\left[\pi^{T}(Q, b)\right]=\$ 1187101$.

\section{Sensitivity Analysis}

Sensitivity analysis has been performed to study the effect of holding cost $(h)$, shortage cost $\left(C_{2}\right)$, expected number of imperfect quality items $(E[\alpha])$, and permissible delay $(M)$ on the lot size $\left(Q^{*}\right)$, backorders $\left(b^{*}\right)$ and the retailer's expected total profit $\left(E\left[\pi^{T}\left(Q^{*}, b^{*}\right)\right]\right)$. (See Tables 1-5).

\section{Table 1}

Effect of changing the permissible delay in payments and shortage cost on optimal order level, backorder level and retailer's expected profit. For $\alpha=0.02$ and $C_{2}=\$ 15 /$ unit/year.

\begin{tabular}{ccccc}
\hline $\boldsymbol{M}$ & $\boldsymbol{C}_{\mathbf{2}}$ & $\boldsymbol{Q}$ & $\boldsymbol{b}$ & Profit \\
\hline \multirow{2}{*}{2} & 5 & 958 & 247 & 2459842 \\
& 10 & 911 & 84 & 2459254 \\
& 20 & 904 & 0 & 2459127 \\
\hline \multirow{2}{*}{5} & 5 & 934 & 264 & 2461740 \\
& 10 & 890 & 97 & 2461571 \\
\hline \multirow{2}{*}{8} & 20 & 874 & 0 & 2461381 \\
& 5 & 930 & 265 & 2464260 \\
& 10 & 880 & 101 & 2463587 \\
\end{tabular}

Table 2

Effect of changing the percentage of defectives on the optimal order level, backorder level, and retailer's expected profit.

\begin{tabular}{cccc}
\hline $\boldsymbol{\alpha}$ & $\boldsymbol{Q}$ & $\boldsymbol{b}$ & Profit \\
\hline 0.01 & 687 & 96 & 2457708 \\
0.02 & 691 & 93 & 2454814 \\
0.03 & 695 & 91 & 2451860 \\
0.04 & 699 & 88 & 2448843 \\
0.05 & 704 & 86 & 2445763 \\
0.06 & 708 & 83 & 2442617 \\
0.08 & 716 & 78 & 2436118 \\
0.1 & 725 & 73 & 2429329 \\
\hline
\end{tabular}

\section{Table 3}

Effect of changing the shortage cost and holding cost on the optimal order level, backorder level, and retailer's expected profit.

\begin{tabular}{ccccc}
\hline $\boldsymbol{C}_{\mathbf{2}}$ & $\boldsymbol{h}$ & $\boldsymbol{Q}$ & $\boldsymbol{b}$ & Profit \\
\hline \multirow{3}{*}{5} & 5 & 1213 & 155 & 2461164 \\
& 10 & 976 & 249 & 2459127 \\
& 15 & 853 & 286 & 2457623 \\
\hline \multirow{2}{*}{8} & 5 & 1189 & 25 & 2460998 \\
& 10 & 937 & 131 & 2458692 \\
\hline \multirow{2}{*}{12} & 15 & 813 & 182 & 2457025 \\
& 5 & 1188 & 0 & 2460992 \\
& 10 & 918 & 43 & 2458465 \\
\hline
\end{tabular}




\section{Managerial Insights}

(i) From Table 1 we can study the interactions between permissible delay in payments and shortages. It indicates that as the shortage cost increases, optimal order level decreases, backorder level decreases, and retailer's expected profit decreases significantly. Also when the shortage cost is high it results in no backorders and very low profits, signifying that model with shortages is better that that of no shortage model. Further ahead, for a fixed shortage cost as the permissible delay in payment increases, optimal order level decreases, backorder level increases and retailer's expected profit increases significantly. Thus, allowing delay in payment exaggerates the benefits obtained from backordered shortages.

(ii) It is clearly evident from Table 2 that as the percentage of defective items increases the optimal order quantity increases but the profit decreases significantly. As the defectives are increasing in the ordered lot, there are more items that are salvaged at a discounted price, resulting in lower profits.

(iii) Table 3 establishes a trade-off between holding and shortage cost. As the holding cost increases, the backorder level increases and optimal order level decreases resulting in lower profits. It is advisable to stock less in the inventory in this case, and backorder more of the demand so as to avoid large holding costs. However, for a fixed holding cost as the shortage cost increases, optimal order level, backorder level and retailer's expected profit decreases significantly.

These observations entail that an inventory model that jointly incorporates shortages and delay in payment performs better than others that incorporate them separately.

\section{Conclusion}

Trade credit has been widely used by many business organizations to promote their trade. It helps in smooth and continuous functioning of trade industry by relaxing the immediate payment of the purchased lot. The received lot may contain a fraction of imperfect quality items, so a screening process is employed by the retailer, so as to serve good quality products to his customers. Thus an inventory model is proposed for deteriorating imperfect quality items under credit policy, where the retailer receives a certain period by the supplier to make the payments. The model also allows for the backorders, and it is assumed that the backordered demand is met right after screening is done on replenished quantity. An algorithm has been employed which jointly optimizes the order quantity and the backorder level, so as to maximize the profit. The model has been validated with the help of a numerical example, and sensitivity analysis with respect to various model parameters has also been presented. The model establishes a trade-off between holding cost and shortage cost. Findings clearly suggest that the presence of trade credit has got affirmative effect on retailer ordering policy.

Further, the proposed model can be extended for more realistic situations such as stochastic and stock dependent demand with inflation etc.

\section{Acknowledgement}

The authors would like to thank anonymous referees for their constructive comments. The first and last author would like to acknowledge the support of the Research Grant No.: RC/2014/6820, provided by the University of Delhi, Delhi, India for accomplishing this research.

\section{Appendix A}

$$
X_{11}=-\frac{2 C_{2} b D}{\lambda Q(1-E[\alpha])}, \quad X_{12}=-\frac{c\left(-\frac{D X_{13}}{\theta X_{14}}+1\right) D}{Q(1-E[\alpha])}, \quad X_{13}=\left(1-E[\alpha] X_{23}-\frac{E[\alpha] Q \theta X_{23}}{\lambda}-\frac{b X_{23}}{\lambda}\right),
$$




$$
\begin{aligned}
& X_{14}=\left(\frac{D}{\theta}+Q-E[\alpha] Q X_{23}-b X_{23}\right), X_{15}=h D\left(\frac{D\left(\frac{X_{22}}{\lambda}-\frac{1}{\lambda}\right)}{\theta Q(1-E[\alpha])}+\frac{X_{22}}{\lambda(1-E[\alpha])}+\frac{D\left(\frac{-\frac{-X_{13} e^{-X^{21}}+\frac{\theta X_{22}}{\lambda}}{X_{14}}-\frac{X_{13}}{\theta X_{14}}+\frac{1}{\lambda}}{\theta}\right)}{\theta Q(1-E[\alpha])},\right. \\
& \left.X_{16}=-\frac{-\frac{X_{13} e^{-X_{21}}}{X_{14}}+\frac{\theta X_{22}}{\lambda}}{\theta(1-E[\alpha])}, X_{17}=\frac{E[\alpha]\left(-\frac{X_{13}}{\theta X_{14}}+\frac{1}{\lambda}\right) e^{\theta\left(-\frac{X_{21}}{\theta}+\frac{Q}{\lambda}\right)}}{1-E[\alpha]}, X_{18}=\frac{b\left(-\frac{X_{13}}{\theta X_{14}}+\frac{1}{\lambda}\right) e^{\theta\left(-\frac{X_{21}}{\theta}+\frac{Q}{\lambda}\right)}}{Q(1-E[\alpha])}\right), \\
& X_{19}=D\left(\frac{-\frac{-X_{13} e^{-X_{21}}+\frac{\theta X_{22}}{\lambda}}{X_{14}}}{\theta}-\frac{X_{13}}{\theta X_{14}}+\frac{1}{\lambda}\right) \\
& X_{21}=\ln \left(\frac{D}{\theta}+Q-E[\alpha] Q X_{23}-b X_{23}\right)+\ln \left(\frac{D}{\theta}\right), X_{22}=e^{-\frac{\theta Q}{\lambda}}, X_{23}=e^{\frac{\theta Q}{\lambda}}, \\
& X_{31}=-\frac{C_{2}\left(\frac{b}{D}+\frac{2 Q}{\lambda}\right) D}{Q(1-E[\alpha])}-\frac{C_{2} b}{Q(1-E[\alpha])}+\frac{c D^{2}\left(\frac{X_{23}}{\theta X_{14}}+\frac{1}{D}\right)}{Q(1-E[\alpha])} \\
& X_{32}=h D\left(\frac{D\left(-\frac{X_{23} \cdot e^{-X_{21}}}{\theta X_{14}}+\frac{X_{23}}{\theta X_{14}}\right)}{\theta Q(1-E[\alpha])}-\frac{X_{23} \cdot e^{-X_{21}}}{\theta X_{14}(1-E[\alpha])}+\frac{E[\alpha] X_{23} \cdot e^{\theta\left(\frac{-X_{21}}{\theta}+\frac{Q}{\lambda}\right)}}{\theta X_{14}(1-E[\alpha])}-\frac{1-e^{\theta\left(\frac{-X_{21}}{\theta}+\frac{Q}{\lambda}\right)}}{\theta Q(1-E[\alpha])}+\frac{b \cdot X_{23} \cdot e^{\theta\left(\frac{-X_{21}}{\theta}+\frac{Q}{\lambda}\right)}}{\theta Q X_{14}(1-E[\alpha])}\right), \\
& X_{33}=c I_{p} D\left(\frac{D\left(-\frac{X_{23} \cdot e^{-X_{21}}}{\theta X_{14}}+\frac{X_{23}}{\theta X_{14}}\right)}{\theta Q(1-E[\alpha])}-\frac{Q X_{23} \cdot e^{-X_{21}}}{X_{14} \theta Q(1-E[\alpha])}+\frac{E[\alpha] Q X_{23} \cdot e^{\theta\left(\frac{-X_{21}}{\theta}+\frac{Q}{\lambda}\right)}}{X_{14} \theta Q(1-E[\alpha])}-\frac{1-e^{\theta\left(\frac{-X_{21}}{\theta}+\frac{Q}{\lambda}\right)}}{\theta Q(1-E[\alpha])}+\frac{b X_{23} \cdot e^{\theta\left(\frac{-X_{21}}{\theta}+\frac{Q}{\lambda}\right)}}{X_{14} \theta Q(1-E[\alpha])}\right) .
\end{aligned}
$$

\section{Appendix B}

$$
\begin{aligned}
& Y_{11}=X_{11}+X_{12} \text {, } \\
& Y_{12}= \\
& \frac{h D}{Q(1-E[\alpha])}\left[\begin{array}{c}
\frac{D\left(\frac{X_{22}}{\lambda}-\frac{1}{\lambda}\right)}{\theta}-\frac{X_{22}-1}{\theta}+\frac{Q X_{22}}{\lambda}+X_{19}-\frac{e^{-X_{21}-X_{22}}}{\theta}-\frac{Q}{\theta}\left(\frac{-X_{13} e^{-X_{21}}}{X_{14}}+\frac{\theta X_{22}}{\lambda}\right) \\
-\frac{E[\alpha]\left(1-e^{\theta\left(-\frac{X_{21}}{\theta}+\frac{Q}{\lambda}\right)}\right)}{\theta}+E[\alpha] Q\left(-\frac{X_{13}}{\theta X_{14}}+\frac{1}{\lambda}\right) \cdot e^{\theta\left(-\frac{X_{21}}{\theta}+\frac{Q}{\lambda}\right)}+b\left(-\frac{X_{13}}{\theta X_{14}}+\frac{1}{\lambda}\right) \cdot e^{\theta\left(-\frac{X_{21}}{\theta}+\frac{Q}{\lambda}\right)}
\end{array}\right], \\
& Y_{13}=c I_{p} D\left[\frac{D\left[\frac{X_{13} e^{-X_{21}}}{\theta X_{14}}-\frac{X_{13}}{\theta X_{14}}\right]}{\theta Q(1-E[\alpha])}+\frac{X_{13} e^{-X_{21}}}{\theta X_{14}(1-E[\alpha])}-\frac{E[\alpha] X_{13} \cdot e^{\left(-\frac{X_{21}}{\theta}+M\right)}}{\theta X_{14}(1-E[\alpha])}-\frac{b X_{13} e^{\left(-\frac{X_{21}}{\theta}+M\right)}}{\theta Q X_{14}(1-E[\alpha])},\right. \\
& Y_{21}=X_{31}-X_{32} \text {, } \\
& Y_{22}=c I_{p} D\left[\frac{D\left[-\frac{X_{23} \cdot e^{-X_{21}}}{\theta X_{14}}+\frac{X_{23}}{\theta X_{14}}\right]}{\theta Q(1-E[\alpha])}-\frac{X_{23} \cdot e^{-X_{21}}}{\theta X_{14}(1-E[\alpha])}+\frac{E[\alpha] X_{23} e^{\theta\left(-\frac{X_{21}}{\theta}+M\right)}}{\theta X_{14}(1-E[\alpha])}-\frac{1-e^{\theta\left(-\frac{X_{21}}{\theta}+M\right)}}{\theta Q(1-E[\alpha])}+\frac{b X_{23} e^{\theta\left(-\frac{X_{21}}{\theta}+M\right)}}{\theta X_{14} Q(1-E[\alpha])} .\right.
\end{aligned}
$$

\section{Appendix C}

$$
\begin{aligned}
& Z_{11}=Y_{11}-Y_{12}, Z_{12}=\frac{p D I_{e}\left[\frac{D\left(X_{13}\right)\left(M-\frac{X_{21}}{\theta}\right)}{\theta X_{14}}-\frac{b}{\lambda}\right]}{Q(1-E[\alpha])}-\frac{C_{S} E[\alpha] D I_{e}}{\lambda(1-E[\alpha])}, Z_{21}=X_{31}-X_{32}, \\
& Z_{22}=\frac{p D I_{e}\left(-\frac{D X_{23}\left(M-\frac{X_{21}}{\theta}\right)+M-\frac{Q}{\lambda}}{\theta X_{14}}\right)}{Q(1-E[\alpha])} .
\end{aligned}
$$

\section{Appendix D}

Find below second order derivatives of expected total profit functions for each of the case: 
Case 1: $0 \leq M \leq t_{s}$

$$
\begin{aligned}
& \frac{\partial^{2} E\left[\pi_{1}^{T}(Q, b)\right]}{\partial Q^{2}}=U_{12}-U_{13}-U_{14}+U_{15}-U_{16}, \\
& \frac{\partial^{2} E\left[\pi_{1}^{T}(Q, b)\right]}{\partial b^{2}}=U_{21}-U_{22}-U_{23}
\end{aligned}
$$

where

$$
\begin{aligned}
& U_{11}=\left(-\frac{2 E[\alpha] \theta X_{23}}{\lambda}-\frac{E[\alpha] X_{23} Q \theta^{2}}{\lambda^{2}}-\frac{b X_{23} \theta^{2}}{\lambda^{2}}\right), \\
& U_{12}=-\frac{c\left[-\frac{D U_{11}}{\theta X_{14}}+\frac{D X_{13}{ }^{2}}{\theta X_{14}{ }^{2}}\right] D}{Q(1-E[\alpha])} \\
& U_{13}=h D\left[-\frac{D X_{22}}{\lambda^{2} Q(1-E[\alpha])}-\frac{\theta X_{22}}{\lambda^{2}(1-E[\alpha])}+\frac{D}{\theta Q(1-E[\alpha])}\left[-\frac{1}{\theta}\left[-\frac{U_{11 .} e^{-X_{21}}}{X_{14}}+\frac{2 X_{13}^{2} \cdot e^{-X_{21}}}{X_{14}^{2}}-\frac{X_{22} \theta^{2}}{\lambda^{2}}\right]-\right.\right. \\
& \left.\frac{U_{11}}{\theta X_{14}}+\frac{X_{13}^{2}}{\theta X_{14}^{2}}\right] \\
& U_{14}=\frac{-1}{\theta(1-E[\alpha])}\left[-\frac{U_{11} e^{-X_{21}}}{X_{14}}+\frac{2 X_{13}^{2} \cdot e^{-X_{21}}}{X_{14}^{2}} \frac{X_{22} \theta^{2}}{\lambda^{2}}\right], \\
& U_{15}=\frac{E[\alpha] Q\left[-\frac{U_{11}}{\theta X_{14}}+\frac{X_{13}^{2}}{\theta X_{14}^{2}}\right] e^{\theta\left(-\frac{X_{21}}{\theta}+\frac{Q}{\lambda}\right)}}{Q(1-E[\alpha])}+\frac{E[\alpha] Q\left[-\frac{X_{13}}{\theta X_{14}}+\frac{1}{\lambda}\right]^{2} \theta e^{\theta\left(-\frac{X_{21}}{\theta}+\frac{Q}{\lambda}\right)}}{Q(1-E[\alpha])}+\frac{b\left[-\frac{U_{11}}{\theta X_{14}}+\frac{X_{13}^{2}}{\theta X_{14}^{2}}\right] e^{\theta\left(-\frac{X_{21}}{\theta}+\frac{Q}{\lambda}\right)}}{Q(1-E[\alpha])}+
\end{aligned}
$$

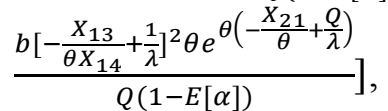

$$
\begin{aligned}
& U_{16}=c I_{p} D\left[-\frac{D X_{22}}{\lambda^{2} Q(1-E[\alpha])}+\frac{2 X_{22}}{\lambda Q(1-E[\alpha])}-\frac{\theta Q X_{22}}{\lambda^{2}(1-E[\alpha])}+\frac{D}{\theta Q(1-E[\alpha])}\left[-\frac{1}{\theta}\left[-\frac{U_{11 . e^{-X_{21}}}}{X_{14}}+\right.\right.\right. \\
& \left.\left.\frac{2 X_{13}^{2} \cdot e^{-X_{21}}}{X_{14}^{2}}-\frac{X_{22} \theta^{2}}{\lambda^{2}}\right]-\frac{U_{11}}{\theta X_{14}}+\frac{X_{13}^{2}}{\theta X_{14}^{2}}\right]-\frac{-\frac{X_{13} \cdot e^{-X_{21}}}{X_{14}}+\frac{X_{13}^{2}}{\theta X_{14}^{2}}}{Q \theta(1-E[\alpha])}-\frac{Q}{\theta Q(1-E[\alpha])}\left[-\frac{U_{11 \cdot e^{-X_{21}}}}{X_{14}}+\frac{2 X_{13}^{2} \cdot e^{-X_{21}}}{X_{14}^{2}}-\right. \\
& \left.\frac{X_{22} \theta^{2}}{\lambda^{2}}\right]+\frac{2 E[\alpha]\left[-\frac{X_{13}}{\theta X_{14}}+\frac{1}{\lambda}\right]^{2} \theta e^{\theta\left(-\frac{X_{21}}{\theta}+\frac{Q}{\lambda}\right)}}{Q(1-E[\alpha])}+\frac{E[\alpha] Q\left[-\frac{U_{11}}{\theta X_{14}}+\frac{X_{13}^{2}}{\theta X_{14}^{2}}\right] e^{\theta\left(-\frac{X_{21}}{\theta}+\frac{Q}{\lambda}\right)}}{Q(1-E[\alpha])}+\frac{\alpha\left[-\frac{X_{13}}{\theta X_{14}}+\frac{1}{\lambda}\right]^{2} \theta e^{\theta\left(-\frac{X_{21}}{\theta}+\frac{Q}{\lambda}\right)}}{Q(1-E[\alpha])}+ \\
& \left.\frac{b\left[-\frac{U_{11}}{\theta X_{14}}+\frac{X_{13}^{2}}{\theta X_{14}^{2}}\right] e^{\theta\left(-\frac{X_{21}}{\theta}+\frac{Q}{\lambda}\right)}}{Q[1-E[\alpha])} \frac{b\left[-\frac{X_{13}}{\theta X_{14}}+\frac{1}{\lambda}\right]^{2} \theta e^{\theta\left(-\frac{X_{21}}{\theta}+\frac{Q}{\lambda}\right)}}{Q(1-E[\alpha])}\right] \text {, } \\
& U_{21}=-\frac{2 C_{2}}{Q(1-E[\alpha])}-\frac{c D^{2} X_{23}^{2}}{\theta X_{14} Q(1-E[\alpha])}, \\
& U_{22}=h D\left[\frac{D\left[-\frac{2 X_{23}^{2} \cdot e^{-X_{21}}}{\theta X_{14}{ }^{2}}+\frac{X_{23}^{2}}{\theta X_{14}{ }^{2}}\right.}{\theta Q(1-E[\alpha])}-\frac{2 X_{23}^{2} \cdot e^{-X_{21}}}{\theta(1-E[\alpha]) X_{14}{ }^{2}}+\frac{2 E[\alpha] X_{23}^{2} e^{\theta\left(-\frac{X_{21}}{\theta}+\frac{Q}{\lambda}\right)}}{\theta(1-E[\alpha]) X_{14}{ }^{2}}+\frac{2 X_{23} e^{\theta\left(-\frac{X_{21}}{\theta}+\frac{Q}{\lambda}\right)}}{\theta Q(1-E[\alpha]) X_{14}}+\right. \\
& \left.\frac{2 b X_{23}^{2} e^{\theta\left(-\frac{X_{21}}{\theta}+\frac{Q}{\lambda}\right)}}{\theta(1-E[\alpha]) X_{14}{ }^{2}}\right] \text {, } \\
& U_{23}=c I_{p} D\left[\frac{D\left[-\frac{2 X_{23}^{2} \cdot e^{-X_{21}}}{\theta X_{14}{ }^{2}}+\frac{X_{23}^{2}}{\theta X_{14}{ }^{2}}\right]}{\theta Q(1-E[\alpha])}-\frac{2 X_{23}^{2} \cdot e^{-X_{21}}}{\theta(1-E[\alpha]) X_{14}{ }^{2}}+\frac{2 E[\alpha] X_{23}^{2} e^{\theta\left(-\frac{X_{21}}{\theta}+\frac{Q}{\lambda}\right)}}{\theta(1-E[\alpha]) X_{14}{ }^{2}}+\frac{2 X_{23} e^{\theta\left(-\frac{X_{21}}{\theta}+\frac{Q}{\lambda}\right)}}{\theta Q(1-E[\alpha]) X_{14}}+\right. \\
& \left.\frac{2 b X_{23}^{2} e^{\theta\left(-\frac{X_{21}}{\theta}+\frac{Q}{\lambda}\right)}}{\theta(1-E[\alpha]) X_{14}^{2}}\right] \text {. }
\end{aligned}
$$

Case 2: $t_{s} \leq M \leq t_{1}$

$$
\frac{\partial^{2} E\left[\pi_{2}^{T}(Q, b)\right]}{\partial Q^{2}}=V_{11}-V_{12},
$$


$\frac{\partial^{2} E\left[\pi_{2}^{T}(Q, b)\right]}{\partial b^{2}}=V_{21}-V_{22}$,

where

$$
\begin{aligned}
& V_{11}=U_{12}-U_{13}-U_{14}+U_{15}, \\
& V_{12}=c I_{p} D\left[\frac{D}{\theta Q(1-E[\alpha])}\left[\frac{U_{11} \cdot e^{-X_{21}}}{\theta X_{14}}-\frac{2 X_{13}^{2} \cdot e^{-X_{21}}}{X_{14}^{2}}-\frac{U_{11}}{\theta X_{14}}+\frac{X_{13}^{2}}{\theta X_{14}^{2}}\right]+\frac{U_{11} \cdot e^{-X_{21}}}{\theta X_{14}(1-E[\alpha])}-\frac{U_{11} \cdot e^{-X_{21}}}{X_{14}^{2} \theta(1-E[\alpha])}-\right. \\
& \frac{E[\alpha] U_{11} \cdot e^{\theta\left(-\frac{X_{21}}{\theta}+M\right)}}{\theta X_{14}(1-E[\alpha])}+\frac{2 E[\alpha]\left(X_{13} \cdot e^{\theta\left(-\frac{X_{21}}{\theta}+M\right)}\right.}{X_{14}^{2} \theta(1-E[\alpha])}-\frac{b U_{11} \cdot e^{\theta\left(-\frac{X_{21}}{\theta}+M\right)}}{\theta X_{14}(1-E[\alpha])}-\frac{2 b\left(X_{13} \cdot e^{\theta\left(-\frac{X_{21}}{\theta}+M\right)}\right.}{X_{14}^{2} \theta(1-E[\alpha])} \\
& V_{21}=U_{21}-U_{22}, \\
& V_{22}=c I_{p} D\left[\frac{D\left[-\frac{2 X_{23}^{2} \cdot e^{-X_{21}}}{\theta X_{14}{ }^{2}}+\frac{X_{23}^{2}}{\theta X_{14}}\right]}{\theta Q(1-E[\alpha])}-\frac{2 X_{23}^{2} \cdot e^{-X_{21}}}{\theta(1-E[\alpha]) X_{14}{ }^{2}}+\frac{2 E[\alpha] X_{23}^{2} e^{\theta\left(-\frac{X_{21}}{\theta}+M\right)}}{\theta(1-E[\alpha]) X_{14}{ }^{2}}+\frac{2 X_{23} e^{\theta\left(-\frac{X_{21}}{\theta}+M\right)}}{\theta Q(1-E[\alpha]) X_{14}}+\right. \\
& \left.\frac{2 b X_{23}^{2} e^{\theta\left(-\frac{X_{21}}{\theta}+M\right)}}{\theta(1-E[\alpha]) X_{14}^{2}}\right] .
\end{aligned}
$$

Case 3: $t_{1} \leq M \leq T$

$$
\begin{aligned}
& \frac{\partial^{2} E\left[\pi_{3}^{T}(Q, b)\right]}{\partial Q^{2}}=W_{11}+W_{12} \\
& \frac{\partial^{2} E\left[\pi_{3}^{T}(Q, b)\right]}{\partial b^{2}}=W_{21}+W_{22} \\
& \text { where } \\
& W_{11}=V_{11} \\
& W_{12}=\frac{p D I_{e}}{Q(1-E[\alpha])}\left[\frac{D U_{11} \cdot\left(M-\frac{X_{21}}{\theta}\right)}{\theta X_{14}}-\frac{D X_{13}^{2} \cdot\left(M-\frac{X_{21}}{\theta}\right)}{\theta X_{14}}-\frac{D X_{13}^{2}}{\theta^{2} X_{14}^{2}}\right] \\
& W_{21}=V_{21} \\
& W_{22}=\frac{p D I_{e}}{Q(1-E[\alpha])}\left[\frac{D X_{23}^{2} \cdot\left(M-\frac{X_{21}}{\theta}\right)}{\theta X_{14}^{2}}-\frac{D X_{23}^{2}}{\theta^{2} X_{14}^{2}}\right]
\end{aligned}
$$

\section{References}

Aggarwal, S. P., \& Jaggi, C. K. (1995). Ordering policies of deteriorating items under permissible delay in payments. Journal of the Operational Research Society, 46, 658-662.

Chakrabarti, T. and Chaudhari, K.S. (1997). An EOQ for deteriorating items with a linear trend in demand and shortages in all cycles. International Journal of production Economics, 49, 205-213.

Chang, H.C., \& Ho, C.H., (2010). Exact closed-form solutions for optimal inventory model for items with imperfect quality and shortage backordering. Omega, 38, 233-237.

Chu, P., Chung, K. J., \& Lan, S. P. (1998). Economic order quantity of deteriorating items under permissible delay in payments. Computers \& Operations Research, 25, 817-824.

Chung, K. J., \& Huang, Y. F. (2006). Retailer's optimal cycle times in the EOQ model with imperfect quality and a permissible credit period. Quality and Quantity, 40, 59-77.

Chung, K. J., \& Ting, P. S. (1994). On replenishment schedule for deteriorating items with timeproportional demand. Production Planning \& Control, 5, 392-396.

Covert, R. P., \& Philip, G. C. (1973). An EOQ model for items with Weibull distribution deterioration. AIIE transactions, 5, 323-326.

Dave, U., \& Patel, L. K. (1981). (T, S i) policy inventory model for deteriorating items with time proportional demand. Journal of the Operational Research Society, 32, 137-142.

Davis, R. A., \& Gaither, N. (1985). Optimal ordering policies under conditions of extended payment privileges. Management Science, 31, 499-509. 
Ghare, P. M., \& Schrader, G. F. (1963). A model for exponentially decaying inventory. Journal of industrial Engineering, 14, 238-243.

Goyal, S. K. (1985). Economic order quantity under conditions of permissible delay in payments. Journal of the operational research society, 36, 335-338.

Hariga, M. (1995). An EOQ model for deteriorating items with shortages and time-varying demand. Journal of the Operational Research Society, 46, 398-404.

Hariga, M. A., \& Benkherouf, L. (1994). Optimal and heuristic inventory replenishment models for deteriorating items with exponential time-varying demand. European Journal of Operational Research, 79, 123-137.

Hollier, R.H. and Mark, K.L. (1983). Inventory replenishment policies for deteriorating items in a declining market. International Journal of Production Research, 21, 813-826.

Jaggi, C. K., Mittal, M., \& Khanna, A. (2013a). Effects of inspection on retailer's ordering policy for deteriorating items with time-dependent demand under inflationary conditions. International Journal of Systems Science, 44, 1774-1782.

Jaggi, C.K., Goel, S.K., \& Mittal, M. (2013b). Credit financing in economic ordering policies for defective items with allowable shortages. Applied Mathematics and Computation, 219, 5268-5282.

Kingsman, B. G. (1983). The effect of payment rules on ordering and stockholding in purchasing. Journal of the Operational Research Society, 34, 1085-1098.

Maddah, B., \& Jaber, M. Y. (2008). Economic order quantity for items with imperfect quality: revisited. International Journal of Production Economics, 112, 808-815.

Maddah, B., Moussawi, L., \& Jaber, M. Y. (2010). Lot sizing with a Markov production process and imperfect items scrapped. International Journal of Production Economics, 124, 340-347.

Moussawi-Haidar, L., Salameh, M., \& Nasr, W. (2014). Effect of deterioration on the instantaneous replenishment model with imperfect quality items. Applied Mathematical Modelling, 38, 59565966.

Papachristos, S., \& Konstantaras, I. (2006). Economic ordering quantity models for items with imperfect quality. International Journal of Production Economics, 100, 148-154.

Porteus, E. L. (1986). Optimal lot sizing, process quality improvement and setup cost reduction. Operations Research, 34, 137-144.

Raafat, F.F., Wolfe, P.M., \& Eldin, H.K. (1991). An inventory model for deteriorating items. Computers \& Industrial Engineering, 20, 89-84.

Rosenblatt, M. J., \& Lee, H. L. (1986). Economic production cycles with imperfect production processes. IIE transactions, 18, 48-55.

Ross, S.M. (1996). Stochastic Processes. $2^{\text {nd }}$ Edition Wiley, New York, NY.

Sachan, R. S. (1984). On (T, S i) policy inventory model for deteriorating items with time proportional demand. Journal of the Operational Research Society, 35, 1013-1019.

Salameh, M. K. (1999). Effect of deteriorating items on the instantaneous replenishment model. Production Planning \& Control, 10, 175-180.

Salameh, M. K., \& Jaber, M. Y. (2000). Economic production quantity model for items with imperfect quality. International Journal of Production Economics, 64, 59-64.

Soni, H., Shah, N. H., \& Jaggi, C. K. (2010). Inventory models and trade credit: a review. Control and Cybernetics, 39, 867-882.

Wee, H.M., Yu, J., \& Chen, M.C. (2007). Optimal inventory model for items with imperfect quality and shortage backordering. Omega, 35, 7-11. 